\title{
Guest Reviewers 1st July, 1989 to 30 June, 1990
}

The Editorial Board acknowledges, with thanks, the assistance of the following guest reviewers:

Ali, M. / LONDON

Allen, G. / OTTAWA

Archer, David P. / Calgary

Ashbury, Edwin L. / KINGSTON

Belisle, Sylvain / MONTRÉAL

Belo, Susan / TORONTO

Bevan, Joan C. / MONTRÉAL

Biehl, Diane R. / WINNIPEG

Bissonnette, Bruno / TORONTO

Blackstock, D. / VANCOUVER

Blaise, Gilbert A. / MONTRÉAL

Blanc, Victor / MONTRÉAL

Bond, David M. / ST. JOHN's

Bose, D. / WINNIPEG

Brown, Hugh / KINGSTON

Brown, Karen A. / TORONTO

Burrows, Frederick A. / TORONTO

Bussieres, Jean S. / QUEBEC CITY

Cannon, John E. / WINNIPEG

Charest, Jean / MONTRÉAL

Chartrand, Daniel / MONTrÉAL

Chong, M. / edmonton

Chung, Frances F.T. / TORONTO

Couture, Jacques / MONTRÉAL

Cowie, Neil / SASKATOON

Cox, Robin G. / Calgary

Crosby, Edward T. / OTTAWA

Cunningham, Anthony J. / DUBLIN, IRELAND

Curran, Michael / otTawa

Davies, Jan A. / CALGARY

Douglas, Joanne M. / VANCOUVER

Doyle, John / TORONTO
Drolet, Pierre / MONTRÉal

Duke, Peter / WINNIPEG

Dwane, Peter D. / KINGSTON

Eagle, C.J. / CALGARY

Edelist, Gerald / TORONTO

Etches, Richard / EDMONTON

Finegan, Barry A. / EDMONTON

Finucane, Brendan T. / EDMONTON

Flanagan, M. L. / VANCOUvER

Flynn, J. / ST. JOHN's

Forrest, J.B. / HAMILTON

Fox, Gordon S. / MONTRÉal

Froese, Alison B. / KIngston

Garnett, R.L. / OTTAWA

Gelb, A. / LONDON

Goresky, Gerald V. / CaLGarY

Grant, Raymer P. / VANCOUVER

Guslits, B. / TORONTO

Ha, Hang / SASKaTOON

Halpern, Stephen H. / TORONTo

Henderson, Jane / MONTREAL

Hew, E. / TORONTO

Hope, Charles E. / Halifax

Janzen, James / CALGARY

Kehler, Chris H. / WINNIPEG

Keith, Ian / SAINT JOHN

Kitts, John B. / OTrAWA

Kleiman, Simcha J. / MONTRÉAL

Lang, S. / SASKATOON

Lee, Richard J. / LONDON

Lerman, Jerrold / TORONTO

Leung, P. / TORONTO 
Lewis, Geraint W. / OTTAWA

Lui, Anne / OtTawa

MacIntosh, Brian R. / CALGary

MacManus, B. / HALIFAX

McIntyre, R. / OTTAWA

McLeod, M.E. / TORONTO

McMorland, Graham H. / vancouver

Mallon, Joseph S. / TORONTO

Malm, David N. / VANCOUVER

Manninen, Pirjo / LONDON

Martineau, Raymond / orTawA

Morrison, James D. / HALIFAX

Murkin, John M. / LONDON

Mutch, W.A.C. / WINNIPEG

Nathan, H.J. / OTTAWA

O'Connor, J.P. / VANCOUVER

Ong, B.Y. / WINNIPEG

Organowski, S. / SASKATOON

Papworth, David / SASKatoon

Penning, J. / OtTawa

Perreault, Claude / MONTRÉAL

Pietak, S. / Kingston

Pytka, Saul / HALIFAX

Quance, Daniel R. / MONTRÉAL

Ralley, Fiona E. / MONTrÉaL

Redfern, Paul A. / ST. JOHN's
Rhine, E. / OTtawa

Robblee, J. / OTTAWA

Roberts, David / OTTAWA

Robinson, Richard / MONTRÉAL

Rogers, K. / TORONTO

Rose, Keith / TORONTO

Samson, B. $/$ OTTAWA

Sandler, Alan N. / TORONTO

Scott, W. A. C. / MONTREAL

Sharpe, Michael / LONDON

Shelley, Eric S. / KINGSTON

Simpson, T. / KINGSTON

Slinger, Peter / MONTRÉAL

Sloan, Paul A. / MONTRÉal

Smith, J. Bruce / MONTRÉAL

Soder, C.M. / halifaX

Spence, Desmond G. / MONTREAL

Stewart, John / CalgarY

Tetrault, Jean-Pierre / SHERBROOKE

Thistlewood, J.M. / CALGaRY

Thomson, Ian R. / WINNIPEG

Trop, Davy / MONTRÉAL

Weeks, Sally K. / MONTRÉAL

Wexler, Howard / LONDON

Wynands, J.E. / OTTAWA 